\title{
The Association of a Floating Elbow and a Homolateral Dislocated Floating Shoulder (About a Case)
}

\author{
El Hacen Sidi, Zakaria Rkiba, Charafeddine El Kassimi, Mohamed Rafai, and Abdelhak Garch
}

\section{ABSTRACT}

The floating elbow is a rare lesion, characterized by the presence of a fracture of the humerus and one or both bones of the homolateral forearm. Double disturbances of the upper suspensory shoulder complex, commonly referred to as "floating shoulder" injuries, are ipsilateral fractures of the clavicle and scapula with loss of bone attachment of the glenoid. The combination of a floating elbow and floating shoulder is extremely rare, added an ipsilateral dislocation of the glenohumeral remains exceptional. No case has been reported in the literature of such a lesional association. This observation makes it possible to describe an extremely rare lesion, by proposing the therapeutic attitude and the prognosis.

Keywords: Floating shoulder; Floating elbow; glenohumeral dislocation, homolateral.

Published Online: January 25, 2021

ISSN: 2593-8339

DOI:10.24018/ejmed.2021.3.1.666

Dr Sidi El Hacen*

Université Hassan 2, CHU Ibn Rochd, Casablanca, Morocco.

(e-mail: drsidielhacen@gmail.com)

Dr Rkiba Zakaria

Dr El Kassimi Charafeddine

Pr Rafai Mohamed

Pr Garch Abdelhak

Université Hassan 2, CHU Ibn Rochd, P32, Casablanca, Morocco.

*Corresponding Author

\section{INTRODUCTION}

The floating elbow is a rare lesion characterized by the presence of a fracture of the humerus and one or both bones of the homolateral forearm [1]. The floating shoulder is also known as the double disturbance of the upper suspensory shoulder complex, associated with ipsilateral fractures of the clavicle and scapula with loss of the glenoid bone attachments [2]. Its association with a floating shoulder with ipsilateral dislocation of the glenohumeral is even rarer. The first description of the floating elbow was reported in 1940 by Winderman [3], and that of the floating shoulder in 1975 by Ganz [4]. Similarly, ipsilateral shoulder dislocation is a very rare lesion; only a few exceptional cases have been reported in the literature. No case has been reported in the literature of an association of a floating elbow and floating shoulder with ipsilateral glenohumeral dislocation.

We report one case of fracture of the clavicle, humerus and radius associated with a dislocation of the homolateral glenohumeral resulting from a high-energy mechanism.

\section{Clinical CASE}

H. A., 61 years old, is a farmer by profession, righthanded, previously autonomous and without antecedents. $\mathrm{He}$ is admitted to the emergency room for a staged trauma of the right upper limb, a cranial and cervical trauma, following an accident on the public highway (ejection from a vehicle). The patient was admitted to the emergency room for intensive care where he was given a check-up and a radiographic check-up.
On admission, the patient was conscious, hemodynamically and respiratorily stable. Examination of the upper limb revealed a deformity of the entire right upper limb with significant oedema, an external axe blow and an enlargement of the anteroposterior diameter of the shoulder. On palpation, the glenoid was found to be empty, with no cutaneous opening, vascular disorder, or nervous deficit, depending on the brachial plexus elements.

The standard radiographic workup showed a subluxation of the $\mathrm{C} 2$, a fracture of the middle third of the right clavicle, an antero-medial shoulder dislocation, a fracture of the humeral diaphysis and a joint fracture of the distal radius, it is a floating elbow according to the classification of Agarval and Chadha [1], defining a flattened elbow and floating shoulder associated with a glenohumeral dislocation (Fig. 1-3).

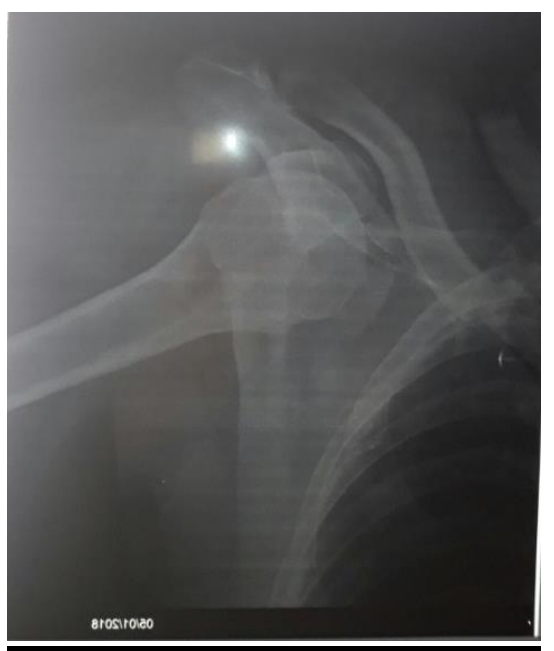

Fig 1. Floating shoulder associated with a glenohumeral dislocation. 


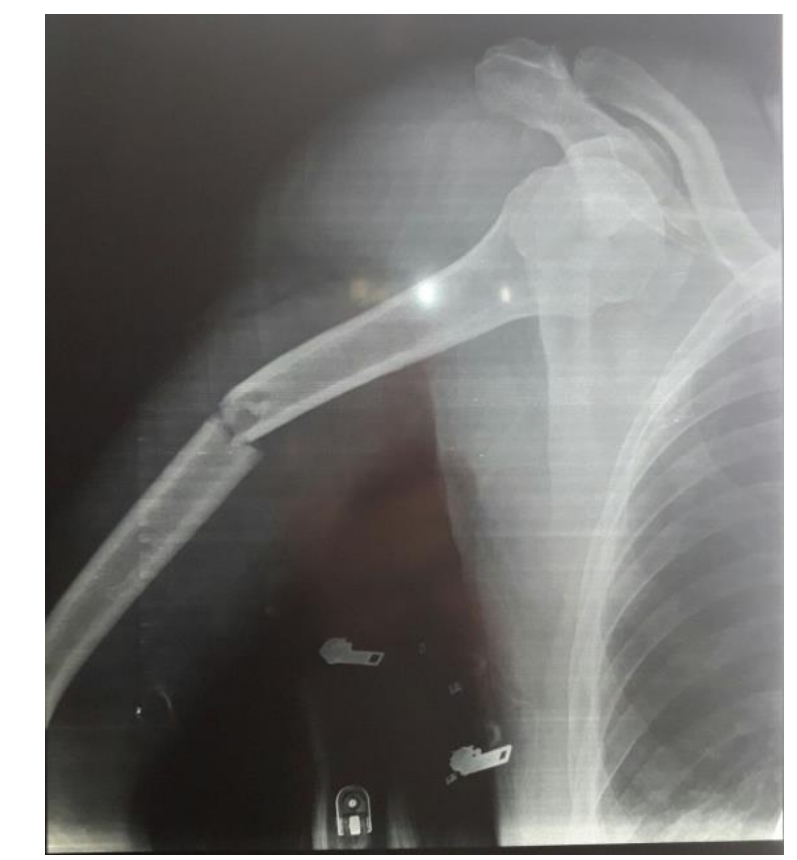

Fig. 2. Antero-medial shoulder dislocation and a fracture of the humeral diaphysis.

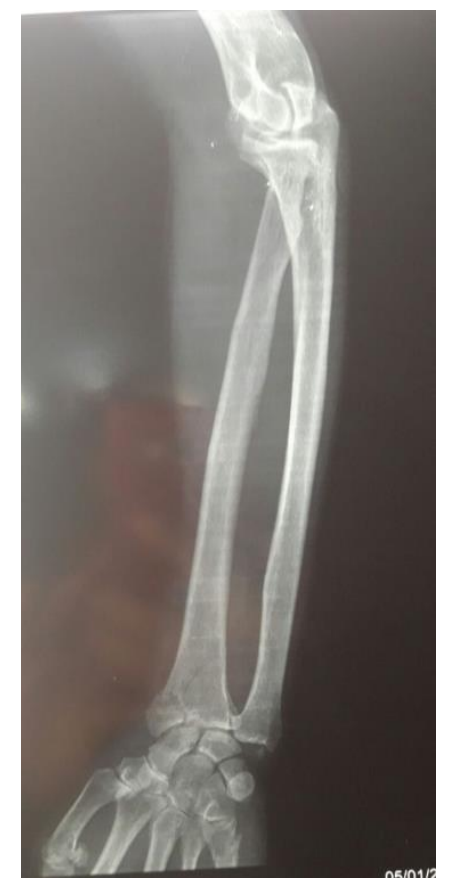

Fig. 3. Fracture of the distal radius.

Cervical spine CT revealed a non-displaced axis fracture with C1-C2 subluxation. The shoulder CT showed an associated fracture displaced of coracoid process type 3 ; that is, a lesion of the shoulder suspensor complex, and pure glenohumeral dislocation (Fig. 4).

After hemodynamic stabilization of the patient, we proceeded to immobilization of the spine by a neck brace and emergency reduction under anesthesia of the shoulder dislocation by Kocher's maneuver.

In the operating room, under general anaesthesia, the patient was placed in supine position on an ordinary table. Initially we performed osteosynthesis of the clavicle with a third tube plate, then of the humerus by static centromedullary nailing, finally we fixed the distal radius with a locked anterior plate Fig $5(\mathrm{a}, \mathrm{b})$. The patient was discharged at day 4 postoperatively with immobilization of the shoulder by an elbow-to-body orthosis for 21 days and rehabilitation was started at week 3 . The neck brace was removed the sixth week, preceded by an X-ray check-up.

The evolution after six months of follow-up was towards the consolidation of all fractures with a limitation of the amplitudes of the right shoulder with a constant score of 75 in relation to the left side. The result was considered good for the floating elbow according to the classification of Lange and Foster [2]. The cervical spine fracture consolidated without neurological sequelae.

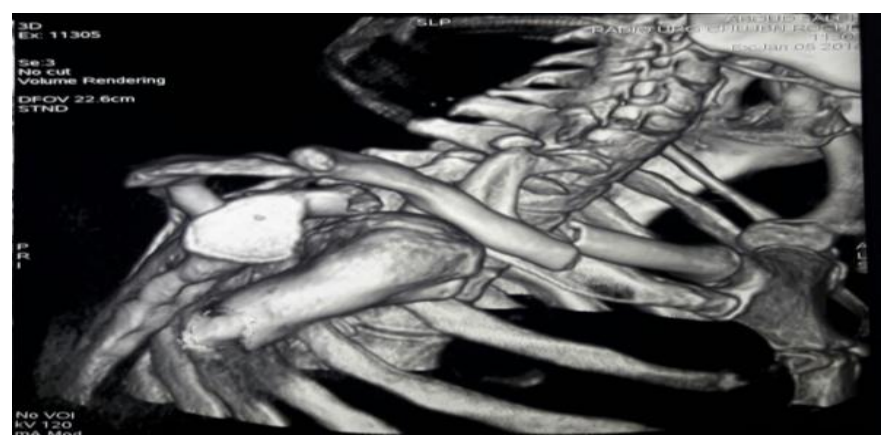

Fig. 4. Shoulder CT lesion of the shoulder suspensor complex, and pure glenohumeral dislocation and fracture displaced of coracoid process.

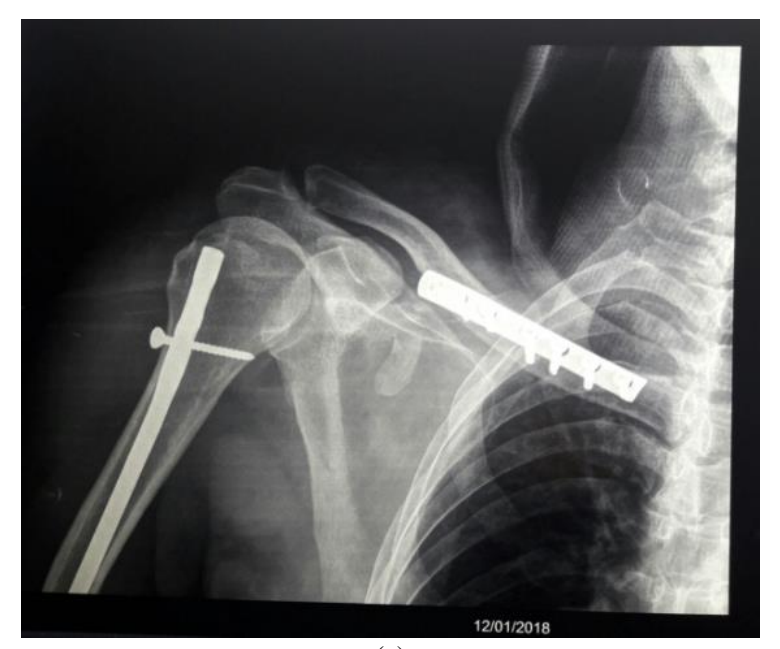

(a)

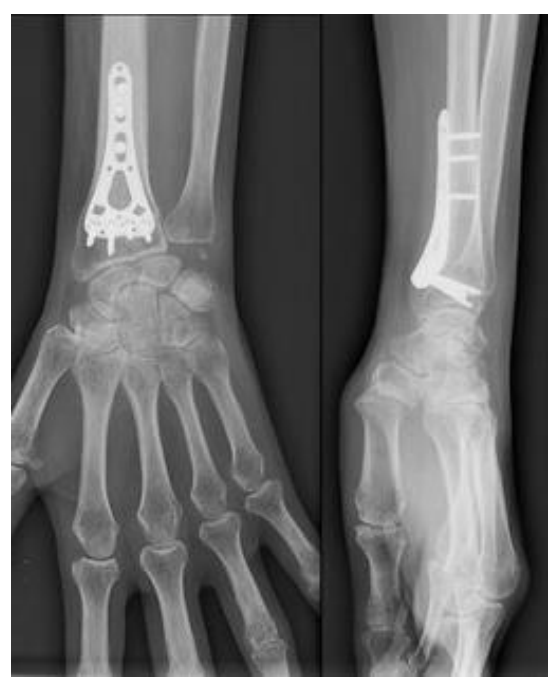

(b)

Fig. 5 (a, b). Osteosynthesis of the clavicle with a third tube plate, of the humerus by static centromedullary nailing, finally we fixed the distal radius with a locked anterior plate. 


\section{DISCUSSION}

The term floating shoulder currently refers to a set of lesions known as double disturbances of the upper suspensory shoulder complex, which are ipsilateral fractures of the clavicle and scapula with loss of bone attachment of the glenoid [3]. The superior suspensory shoulder complex (CSSS) is a ring of bone-soft tissue consisting of the glenoid, coracoid and acromion processes, as well as the distal aspect of the clavicle and their corresponding ligaments; the acromioclavicular joint also belongs to this structure [4].

Homolateral fractures of the humerus and of one or both forearm bones, known generically as "floating elbows", are a lesional association that is uncommon in traumatology [5].

The combination of a dislocated floating shoulder and a homolateral floating elbow is an extremely rare entity [6], the mechanism is a high-energy shock. Several rare lesions have been reported such as a floating dislocated elbow with ipsilateral shoulder dislocation described by Bettuzzi [7], or from a floating elbow to a monteggia lesion or elbow dislocation [8], or even a dislocated shoulder [9]. But no case of lesional association like ours has been described.

The first description of the floating elbow was reported in 1940 by Winderman [4], and that of the floating shoulder in 1975 by Ganz and Noesbergerl, then in 1993 Goss [5] introduced the notion of the double perturbation of the upper suspensory shoulder complex, which can cause high instability.

It was extremely difficult to determine the exact mechanism of the trauma. The force transmitted through the elbow in flexion and the shoulder in abduction and extension resulted in multiple staggered injuries following the shock transmission wave.

In the literature, the various authors recommend concomitant treatment of the lesions with rigid internal osteosynthesis allowing early rehabilitation. For the shoulder suspensor complex lesion, fixation of the clavicle alone has been reported with good functional results [10]. While some authors advocate osteosynthesis of all lesions with early rehabilitation [7], [8].

In our case, data concerning management and recoil are not available due to the rarity of the lesion. Complications of high-energy trauma to the upper limb are primarily of nervous vascular, cutaneous, pseudarthrosis and stiffness according to Rogers et al [11] and Yokohama [12].

\section{CONCLUSION}

The floating shoulder is a rare lesion formerly described by the association of a fracture and the neck of the scapula, currently defined by the disruption of the shoulder suspensory complex. Its association with a floating elbow and ipsilateral glenohumeral dislocation has never been described to our knowledge.

In this article, we report a case of a lesional entity association of a dislocated floating shoulder and a homolateral floating elbow resulting from a high-energy mechanism. Complications remain frequent, sometimes serious given the importance of the trauma. The reduction of the dislocation and the immediate and stable fixation of the fractures in a single operation, as well as early rehabilitation, is the only guarantee of good long-term results.

\section{REFERENCES}

[1] A. Agarwal, M. Chadha, "Floating injuries: a review of the literature and proposal for a universal classification", Acta Orthop Belg, vol. 70, pp. 509-514, 2004.

[2] R. H. Lange, R. J. Fostern, "Skeletal management of humeral shaft fractures associated with forearm fractures", Clin Orthop, vol. 195, pp.173-177, 1985.

[3] Jose B. Toro, David L. Helfet, "Surgical Management of the Floating Shoulder Techniques", Shoulder and Elbow Surgery, Vol. 5, Issue 2, pp. 116-121, 2004.

[4] Heng K1. "Floating shoulder injuries", Int J Emerg Med., 9(1):13, Dec 2016.

[5] J. Bartoníček, M. Tuček, \& O. Naňka, "Floating Shoulder", JBJS Reviews,2018, 1. doi:10.2106/jbjs.rvw.17.00198.

[6] K. Gilde,M. Hoffmann, "Functional outcomes of operative fixation of clavicle fractures in patients with floating shoulder girdle injuries", Journal of Orthopaedics and Traumatology, vol. 16, pp. 221-227, 2015.

[7] C. Bettuzzi, M. Cappuccio, F Cuoghi, D. Tigani,"Floating dislocated elbow with ipsilateral shoulder dislocation: double dislocation upper arm", J Orthop Sci., vol. 20(4), pp. 761-764, Jul2015.

[8] H. B. Solomon, M. Zadnik, W. Eglseder, "A review of outcomes in 18 patients with floating elbow", J Orthop Trauma, vol. 17, pp. 56370, 2003.

[9] K. Ditsios, A. Boutsiadis, P. Papadopoulos, D. Karataglis, "Floating elbow injuries in adults: prognostic factors affecting clinical outcomes", Journal of Shoulder and Elbow Surgery, vol. 22(1), pp. 74-80, 2013. doi:10.1016/j.jse.2012.09.005

[10] H. Hashiguchi, H. Ito, "Clinical outcome of the treatment of floating shoulder by osteosynthesis for clavicular fracture alone", Journal of Shoulder and Elbow Surgery, vol. 12(6), pp. 589-591, 2003. doi:10.1016/s1058-2746(03)00179-4

[11] J. F. Rogers, J. B. Bennet, H. S. Tullos, "Management of concomitant ipsilateral fractures of the humerus and forearm", J Bone Joint Surg. Vol. 66(4), pp. 552-556, 1984.

[12] K. Yokoyama, M. Itoman, A. Kobayashi, M. Shindo, M. Futami "Functional outcomes of floating elbow injuries in adult patients", $J$ Orthop Trauma, vol. 12, pp. 284-290, 1998. 